\title{
Maladies à transmission vectorielle : mettre fin au débat entre déterminants climatiques et déterminants sociaux
}

\author{
Berrang-Ford $\mathrm{L}^{1 *}$, Harper $\mathrm{SL}^{2}$, Eckhardt $\mathrm{R}^{3}$ \\ Affiliations \\ 1 Université McGill, Montréal (Québec) \\ 2 Université de Guelph, Guelph (Ontario) \\ ${ }^{3}$ Département de géographie, Université McGill, Montréal (Québec) \\ *Correspondance : lea.berrangford@mcgill.ca
}

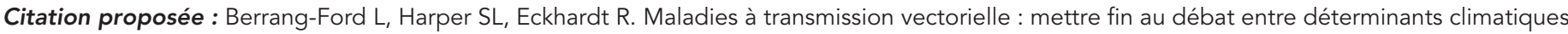
et déterminants sociaux. Relevé des maladies transmissibles au Canada 2016;42:235-6. https://doi.org/10.14745/ccdr.v42i10a09f

\section{Contexte}

Nous avons depuis longtemps reconnu le rôle des changements climatiques et environnementaux comme déterminants de la distribution et de l'incidence des maladies à transmission vectorielle (MTV), aussi bien à l'échelle mondiale qu'au niveau local. Les MTV sont considérées comme particulièrement sensibles aux changements climatiques en raison de l'importance du climat et des conditions météorologiques pour déterminer le cycle de vie des vecteurs de maladies et le potentiel de transmission des pathogènes. Plusieurs de ces changements et facteurs écologiques importants sont eux-mêmes déterminés par le changement social, et la distribution des MTV reste fortement liée aux déterminants sociaux.

Un débat est né dans le domaine des changements climatiques et de la santé concernant l'importance des changements climatiques par rapport aux déterminants sociaux de la santé. Ce débat - attribuable en grande partie à la complexité conceptuelle et méthodologique qui est associée à l'intégration des déterminants climatiques et sociaux aux modèles quantitatifs - a contribué à des points de vue allant de « il y a peu de preuves indiquant que les changements climatiques ont déjà favorisé des maladies infectieuses » (1) à "les changements climatiques représentent est potentiellement la plus grande menace mondiale du XXle siècle pour la santé » (2). Le domaine de la santé publique repose sur la prise de décision fondée sur des preuves. Par conséquent, notre incapacité à conceptualiser de manière adéquate et à modéliser avec certitude les répercussions du climat sur la santé dans le contexte des gradients sociaux existants constitue un obstacle important à une intervention de santé publique efficace en réponse aux changements climatiques.

\section{Objectif}

Passer en revue notre compréhension conceptuelle de la manière dont les variables sociales et climatiques interagissent pour affecter la propagation des MTV.
Évaluer de façon critique dans quelle mesure les approches conceptuelles et les méthodes analytiques actuelles en épidémiologie ont - ou n'ont pas - réussi à intégrer les déterminants sociaux dans les projections de changements climatiques.

Cerner les difficultés et les possibilités liées à une meilleure intégration conceptuelle et méthodologique des déterminants sociaux et climatiques des MTV dans le contexte des changements climatiques.

\section{Narratif}

Trois points clés sont présentés au moyen d'études de cas. a) Les effets des déterminants sociaux sur les MTV sont importants et bien établis, mais mal intégrés aux modèles climatiques. b) Les déterminants climatiques et sociaux interagissent pour affecter la propagation des MTV et se manifestent à des échelles très différentes. Il est donc difficile, pas particulièrement constructif et, dans certains cas, inapproprié de comparer leur effet relatif sur la propagation des MTV. c) En raison de modification sociale des effets du climat, les gradients sociaux existants en santé fournissent des points d'entrée pour les interventions visant à s'adapter aux répercussions annoncées des changements climatiques sur les MTV.

Cela ne signifie pas que les déterminants sociaux sont plus importants que les déterminants écologiques ou climatiques, mais plutôt que les variables sociales sont les facteurs proximaux qui modifieront dans quelle mesure le climat aura des répercussions sur les MTV à l'échelle mondiale. Deux exemples illustrent ces points.

Le premier cas évalue la probabilité d'une réapparition du paludisme autochtone (acquis localement, transmis par les moustiques) au Canada $(3,5)$. La transmission du paludisme a déjà été observée au Canada par le passé, et le sud de l'Ontario présente des conditions (présence d'un vecteur compétent, potentiel de conditions climatiques limitées mais suffisantes pour la réplication du parasite) qui sont propices à sa transmission 
aujourd'hui (3). Plus précisément, le parasite du paludisme, Plasmodium vivax, a besoin d'environ 30 jours à $18^{\circ} \mathrm{C}$ ou 20 jours à $20^{\circ} \mathrm{C}$. Au-dessus de $33^{\circ} \mathrm{C}$ ou en dessous de $16^{\circ} \mathrm{C}$, le cycle ne peut pas être achevé et la transmission n'aura pas lieu (3). Ces conditions se produisent déjà au Canada pendant de courtes périodes et à des endroits où les moustiques vecteurs sont compétents (en particulier au sud de l'Ontario) et, avec le réchauffement à venir, le nombre potentiel de cycles et de réplications devrait augmenter. Par exemple, de 1983 à 2003, on a enregistré moins de 40 jours consécutifs par an où la température quotidienne moyenne était supérieure à $18{ }^{\circ} \mathrm{C}$ dans le sud de l'Ontario. D'ici 2070-2099, on peut s'attendre à 90 jours consécutifs par an où la température sera supérieure à 18 ${ }^{\circ} \mathrm{C}$ dans cette région, ce qui correspond potentiellement à trois cycles complets de transmission de $P$. vivax chez les moustiques canadiens (3). Il s'agit là de sous-estimations, puisque le parasite n'a pas besoin que les jours de chaleur soient consécutifs pour effectuer la transmission. En parallèle, l'augmentation des déplacements internationaux depuis des régions où le paludisme est endémique et les densités de population élevées coïncident spatialement dans le sud de l'Ontario, ce qui accroît la probabilité d'intensité théorique de transmission (3).

Toutefois, une analyse spatiotemporelle à plus petite échelle du risque dans le sud de l'Ontario indique que très peu de cas de paludisme importé (présence du parasite) coïncident avec des régions où les vecteurs sont présents $(4,5)$. Les cas de paludisme importé sont principalement situés dans la région du Grand Toronto, dans des quartiers à faible revenu et à densité de population élevée où vit une proportion plus élevée d'immigrants originaires de régions où le paludisme est endémique (4). En revanche, les vecteurs sont en grande partie présents dans les banlieues de Toronto et les zones périphériques en bordure de la région du Grand Toronto (5). Cette étude de cas illustre à quel point le risque associé aux MTV dépend de l'échelle, et elle montre que les échelles de variation des déterminants sociaux et climatiques - et, en général, de disponibilité des données - sont souvent extrêmement différentes. Sur le plan spatial, alors que les déterminants sociaux varient généralement d'un quartier à l'autre, le climat et les conditions météorologiques ne varient pas entre les quartiers, mais plutôt à une échelle régionale plus vaste. De même, au niveau temporel, les conditions météorologiques peuvent varier d'heure en heure, tandis qu'en général, les déterminants sociaux des MTV ne varient de façon significative que sur plusieurs années ou décennies.

Le cas du paludisme au Canada illustre comment les rôles des déterminants sociaux et climatiques du risque associé aux MTV interagissent et montre à quel point il est difficile de les démêler. Ce point sera précisé ultérieurement en présentant les résultats à venir de l'Ouganda et du Pérou, qui démontrent que les gradients sociaux modifient de façon significative l'effet des conditions météorologiques sur l'incidence des MTV. Dans ces autres exemples, il peut être empiriquement démontré que les variables sociales ne sont pas simplement des facteurs de confusion (moyens de contrôle) des effets du climat sur la santé, mais plutôt que leur effet modifie le degré d'influence du climat sur les MTV. En dépit de cela, les variables sociales sont rarement intégrées aux modèles climat-santé comme facteurs modificateurs de l'effet. Dans ce contexte, nous ne pouvons pas attribuer les changements des MTV uniquement aux variations du climat.

\section{Conclusion}

Face au faible succès des efforts internationaux pour réduire les émissions responsables des changements climatiques, nous devons nous adapter aux répercussions émergentes des changements climatiques sur la santé. L'intervention sur les déterminants climatiques de la santé constitue donc un levier inadéquat, bien qu'important pour les mesures de santé publique. Nous pouvons mettre au point des interventions visant à modifier les déterminants sociaux. En tant que facteurs modificateurs de l'effet de la relation entre le climat et les maladies à transmission vectorielle, les interventions sociales peuvent permettre de réduire les répercussions du climat sur la santé au-delà des bienfaits directs pour la santé en réduisant la vulnérabilité au climat. Les déterminants sociaux de la propagation des MTV constituent la possibilité la plus faisable, et peut-être la seule qui soit réaliste, pour le contrôle et la prévention des MTV dans le contexte des changements climatiques.

\section{Conflit d'intérêts}

Aucun.

\section{Références}

1. Lafferty KD. The ecology of climate change and infectious diseases. Ecology 2009;90:888-900.

2. Costello A, Abbas M, Allen A, Ball S, Bell S, Bellamy R, et al. Managing the health effects of climate change: Lancet and University College London Institute for Global Health Commission. Lancet 2009;373:1693-733.

3. Berrang-Ford L, MacLean JD, Gyorkos TW, Ford JD, Ogden $\mathrm{NH}$. Climate change and malaria in Canada: A systems approach. Interdiscip Perspect Infect Dis 2009;385487.

4. Eckhardt R, Berrang-Ford L, Ross NA, Pillai DR, Buckeridge DL. A spatial analysis of individual- and neighbourhood-level determinants of malaria incidence in adults, Ontario, Canada Emerg Infect Dis 2012; 18:775-82.

5. Eckhardt R. An assessment of imported malaria and the risk of autochthonous transmission in Ontario, Canada. MA thesis, Department of Geography, McGill University, Montréal, Canada 2010. 